\title{
Revealing hub pathway cross-talk for premature newborns with bronchopulmonary dysplasia by the integration of pathway analysis and Monte Carlo Cross-Validation
}

\author{
CHENGBIN WANG* , BIN ZHU*, MING CHEN, GAOYAN CHEN, MUZHEN XU and RUI PAN \\ Department of Pediatrics, Xiangyang Central Hospital, Hubei University of Arts and Science, \\ Xiangyang, Hubei 441021, P.R. China
}

Received July 6, 2018; Accepted January 22, 2019

DOI: $10.3892 /$ etm.2019.7257

\begin{abstract}
The objective of this study was to reveal hub pathway cross-talk for premature newborns with bronchopulmonary dysplasia (BPD) based on the pathway enrichment analysis and Monte Carlo Cross-Validation (MCCV) method. The inference of key pathway cross-talk consisted of four parts: i) Identifying differentially expressed genes (DEGs); ii) detecting differentially expressed pathways (DEPs); iii) computing discriminating score (DS) for each pair of DEPs or cross-talk and investigating seed cross-talk through the random forest (RF) algorithm and iv) extracting hub cross-talk dependent on the MCCV method. The results showed that a total of 132 DEGs and 137 DEPs were obtained across BPD patients and normal controls. Using the DS and RF algorithm, 10 seed DEP cross-talk were detected. By conducting the MCCV on seed cross-talk, 3 hub cross-talk for BPD were uncovered: i) The pair of pathways role of interleukin-17F (IL-17F) in allergic inflammatory airway diseases and role of IL-17A in psoriasis; ii) the pair of pathways role of IL random forest $17 \mathrm{~A}$ in psoriasis and IL-17A signaling in fibroblasts and ii) the pair of pathways IL-17A signaling in airway cells and role of hypercytokinemia/hyperchemokinemia in the pathogenesis of influenza. These 3 hub cross-talk among DEPs might give an insight to reveal the molecular mechanism underlying the premature newborns with BPD.
\end{abstract}

\section{Introduction}

Bronchopulmonary dysplasia (BPD), one of the most common complications of prematurity, is a lung injury in preterm

Correspondence to: Dr Rui Pan, Department of Pediatrics, Xiangyang Central Hospital, Hubei University of Arts and Science, 136 Jingzhou Street, Xiangyang, Hubei 441021, P.R. China

E-mail: ruipanhb@163.com

*Contributed equally

Key words: bronchopulmonary dysplasia, pathway, crosstalk, Monte Carlo Cross-Validation infants resulting from oxygen and mechanical ventilation $(1,2)$. Although progress has been made in neonatal care, the incidence of BPD is still increasing, mainly because the survival rate of younger and more premature babies has increased (3). Hence, effective prevention and treatments for BPD are on urgent need, and their necessary precondition is a comprehensive understanding of molecular and pathological mechanisms underlying this disease.

Previous literature has demonstrated that prenatal factors have important roles in the progression of BPD, such as surfactant deficiency and maternal infection $(4,5)$. Recently, genetic variants were discovered in preterm infants of $\operatorname{BPD}(6,7)$. For instance, mutations of the lipid transporter ATP-binding cassette subfamily A member 3 ( $A B C A 3)$, which is involved in surfactant synthesis in alveolar type II cells, have been revealed in BPD premature newborns (7). Moreover, these studies have highlighted a potential role for molecular pathways involved in inflammation and lung development in affected infants. Cross-talk among gene pathways can be means of regulatory interaction among different pathways or can express the gene overlap among pathways $(8,9)$.

In this study, we proposed to reveal hub pathway cross-talk for premature newborns with BPD based on the integration of pathway related analyses and Monte Carlo Cross-Validation (MCCV) algorithm. Ultimately, hub cross-talk was extracted from seed cross-talk dependent on the MCCV method. The study of cross-talk involved in BPD could help in defining the molecular mechanisms leading to the onset and progression of the pathology (10).

\section{Materials and methods}

Data collection. Gene expression data with accession number E-GEOD-8586 (11) was collected from the ArrayExpress database for premature newborns at risk of BPD related studies. In E-GEOD-8586, there were 54 newborns with a gestational age from 23 to 27 weeks. These 54 newborns included 20 infants who later developed BPD (BPD group) and 34 infants who did not develop BPD (normal controls). There were 23 females and 31 males. Subsequently, in order to control the quality of the data, background corrections and standard normalizations were conducted. 
Identification of differentially expressed genes (DEGs). For investigating the differentially expressed levels of genes across BPD samples and normal controls, the empirical bayes (EB) approach was implemented to identify DEGs. In particular, the EB approach is equivalent to shrinkage of the estimated sample variances towards a pooled estimate, resulting in far more stable inference when the number of arrays is small (12). During this process, $\mathrm{P}$-value for each gene was adjusted by Benjamini-Hochberg (BH) test (13). Importantly, only genes that met the thresholds of false discovery rate (FDR) $<0.01$ and $\mid \log _{2}$ FoldChangel $>2$ simultaneously were considered as DEGs in BPD and normal control groups.

Determination of differentially expressed pathways (DEPs). In the present study, all human biological pathways were derived from the Ingenuity Pathways Analysis (IPA) tool. Subsequently, DEGs of BPD were mapped to these biological pathways with an attempt to make them more correlated with the disease, and we gained pathways that were responsible for coordinating DEG activities, named as background pathways. Then we evaluated the significance between DEGs and genes in background pathways using Fisher's exact test (14). P-values were adjusted by BH test (13). In addition, the cut-off for DEPs across BPD patients and normal controls was $\mathrm{P}<0.01$.

DEP cross-talk investigation. Based on the DEPs, we further focused on the relationships among any two of them. If a correlation existed between two DEPs, the DEP pair would be defined as a cross-talk for DEPs (10). Hence, we selected the discriminating score (DS) to access the strength of cross-talk among DEPs in the present study (10). Supposing that $\mathrm{x}$ and $\mathrm{y}$ were two DEPs in cross-talk, the DS was computed as following formula:

$$
D S(x, y)=\frac{\left|u_{x}-u_{y}\right|}{\sigma_{x}+\sigma_{y}}
$$

Seed cross-talk extraction. With an attempt to evaluate the functional activities of DEP cross-talk more deeply, a random forest (RF) classification model (15) was utilized to access the classified performance of these pairs between BPD and normal control groups. The method was comprised of three sections, first, drawing Ntree bootstrap samples dependent on the DS for each DEP cross-talk $\left(N_{\text {tree }}=500\right)$; secondly, growing a regression tree for each of the bootstrap samples; and finally, aggregating the predictions of the $N_{\text {tree }}$ trees. The top $10 \mathrm{DEP}$ cross-talk in descending order of AUC were defined as seed cross-talk.

MCCV for seed cross-talks. As mentioned above, seed cross-talk was identified, and then the MCCV method was adopted to validate the biological functions of seed cross-talk in BPD samples (16). The 54 samples were randomly split into two sets, the calibration set (Sc), contained nc samples for fitting the models; and the validation set (Sv), included nv samples for validating the model.

$$
\operatorname{MCCV}_{n_{v}}=\frac{1}{R n_{v}} \sum_{k=1}^{R}\|E\|^{2}
$$

Of which E represents the squared prediction error, $R$ represents the procedure repeated times $(R=50)$. Theoretically, the fewer samples used in model calibration, the more repeat times were needed. For each bootstrap, the DEGs, DEPs, cross-talk and their DS values were carried out. The repeat times of DEP cross-talk were statistically calculated. More repeat times might imply more significance of this cross-talk.

\section{Results}

DEGs. In the present study, there were 20,514 genes in the pretreated E-GEOD-8586. After performing EB algorithm, a total of 132 DEGs which satisfied the cut-off $(\mathrm{P}<0.01$ and $\mid \log _{2}$ FoldChangel $>2$ ) between BPD premature newborns and normal controls were obtained. All DEGs were ranked in descending order of their P-values (Table I). The smaller the P-value, the more significant the expression of the DEG was. The most significant five DEGs across BPD group and normal controls were EMILINI $\left(\mathrm{P}=4.29 \mathrm{Ex} 10^{-6}\right)$, MFAP51 $\left(\mathrm{P}=8.77 \mathrm{Ex} 10^{-6}\right), S N C A 1\left(\mathrm{P}=9.23 \mathrm{Ex} 10^{-6}\right)$, interleukin-62 (IL-62) $\left(\mathrm{P}=3.14 \mathrm{Ex} 10^{-5}\right)$ and STRADBI $\left(\mathrm{P}=7.56 \mathrm{Ex} 10^{-5}\right)$.

DEPs. As described above, the IPA tool with 589 biological pathways (covering 5,169 genes) was implemented to execute pathway enrichment analysis for 132 DEGs of BPD infants, and the Fisher's exact test was used to evaluate significance of the pathways. Consequently, a total of 137 DEPs with $\mathrm{P}<0.01$ were identified between BPD samples and normal controls, of which role of IL-17A in psoriasis $\left(\mathrm{P}=5.96 \mathrm{Ex} 10^{-13}\right)$ was the most significant one. Furthermore, DEPs with larger number of DEGs might be more important than that of the smaller ones. The DEPs with DEG number $\geq 4$ are listed in Table II. Among these DEPs, flucocorticoid receptor signaling $\left(\mathrm{P}=7.11 \mathrm{Ex} 10^{-7}\right)$ contains 6 DEGs. Interestingly, the number of DEGs enriched in 6 DEPs was 5, including granulocyte adhesion and diapedesis $\left(\mathrm{P}=1.55 \mathrm{E} \times 10^{-6}\right)$, role of IL-17A in psoriasis $\left(\mathrm{P}=5.96 \mathrm{Ex} 10^{-13}\right)$, glucocorticoid receptor signaling $\left(\mathrm{P}=5.32 \mathrm{Ex} 10^{-6}\right)$, role of IL-17A in arthritis $\left(\mathrm{P}=2.24 \mathrm{E}-10^{-8}\right)$, IL-17A signaling in airway cells $\left(\mathrm{P}=4.54 \mathrm{Ex} 10^{-8}\right)$ and flucocorticoid receptor signaling $\left(\mathrm{P}=4.70 \mathrm{Ex} 10 \times 10^{-5}\right)$.

Seed cross-talk. In this section, first of all, DS was assigned to any pair of DEPs or cross-talk by comparing their gene expression levels. In consequence, 609 cross-talk among 137 DEPs were identified, and the DS distribution is illustrated in Fig. 1. Two of the cross-talk had higher DS than the others: Role of IL-17A in psoriasis; IL-17A signaling in fibroblasts (DS=6.328) and LXR/RXR activation; role of IL-17A in psoriasis (DS=6.134). Subsequently, we applied the RF classification of cross-talk for each sample based on the DS, and chose the AUC to evaluate the classification performance of each cross-talk. The cross-talk was ranked according to AUC in a descending order and the top 10 cross-talk were denoted as seed cross-talks (Table III). The best cross-talk was the pair of pathways acute phase response signaling and gepatic fibrosis/hepatic stellate cell activation (AUC=0.915). Besides, the AUC for the following two cross-talks were ranged from 0.8 to 0.9 : The pair of pathways role of IL-17F in allergic inflammatory airway diseases and role of IL-17A in 
Table I. Differentially expressed genes (DEGs).

\begin{tabular}{|c|c|c|c|c|c|c|c|}
\hline Rank & Genes & Rank & Genes & Rank & Genes & Rank & Genes \\
\hline 1 & EMILINI & 34 & LINC00702 & 67 & TRIM58 & 100 & CXCL3 \\
\hline 2 & MFAP51 & 35 & SLC25A391 & 68 & $B P G M$ & 101 & MORC4 \\
\hline 3 & SNCAl & 36 & $S T R A D B 2$ & 69 & SLC19A2 & 102 & $C 3$ \\
\hline 4 & $I L-62$ & 37 & $X K 1$ & 70 & ZВTB16 & 103 & SERPINE11 \\
\hline 5 & STRADB1 & 38 & EPB 422 & 71 & SNCA & 104 & $N P T N-I T 1$ \\
\hline 6 & CXCL12 & 39 & $A H S P 1$ & 72 & $D M T N$ & 105 & $P T G S 2$ \\
\hline 7 & $B P G M 1$ & 40 & KRT13 & 73 & $E P B 42$ & 106 & CXCL6 \\
\hline 8 & CXCL32 & 41 & S100A91 & 74 & РТХ31 & 107 & $S G K 1$ \\
\hline 9 & TNFAIP32 & 42 & SOD22 & 75 & $I L-61$ & 108 & CXCL8 \\
\hline 10 & SOD21 & 43 & $B O K$ & 76 & $H E M G N$ & 109 & S100A8 \\
\hline 11 & EPB421 & 44 & GYS1 & 77 & KRT1 & 110 & TNFAIP3 \\
\hline 12 & РTX32 & 45 & CCL22 & 78 & $B L V R B$ & 111 & $L I F$ \\
\hline 13 & CCL21 & 46 & S100A81 & 79 & STRADB & 112 & CXCL1 \\
\hline 14 & $H B M 1$ & 47 & KRT141 & 80 & FAM46C & 113 & FOSB1 \\
\hline 15 & CXCL62 & 48 & MFAP52 & 81 & $U C P 2$ & 114 & CCL2O \\
\hline 16 & KRT14 & 49 & ZВTB163 & 82 & SERPINE12 & 115 & $I L-6$ \\
\hline 17 & PLEKHOI & 50 & CCL2O2 & 83 & $X K$ & 116 & SOD2 \\
\hline 18 & $U C P 21$ & 51 & CXCL33 & 84 & $A H S P$ & 117 & TNFAIP6 \\
\hline 19 & KAT2B1 & 52 & HBM4 & 85 & $G C H 1$ & 118 & PTX3 \\
\hline 20 & РОС $1 B$ & 53 & CXCL63 & 86 & CHRDL2 & 119 & S100A9 \\
\hline 21 & ADIPORI & 54 & РTX33 & 87 & $L I F 1$ & 120 & $C C L 2$ \\
\hline 22 & $I D 1$ & 55 & CXCL82 & 88 & CXCL31 & 121 & S100A12 \\
\hline 23 & $H B Q 1$ & 56 & CXCL13 & 89 & TNFAIP61 & 122 & MFAP5 \\
\hline 24 & FAM46Cl & 57 & $S Q R D L$ & 90 & CCL201 & 123 & EGRI \\
\hline 25 & $D C A F 12$ & 58 & MAP $3 K 8$ & 91 & SLC25A39 & 124 & FOS \\
\hline 26 & $B P G M 2$ & 59 & $D C K$ & 92 & $H B M$ & 125 & $F O S B$ \\
\hline 27 & C15orf48 & 60 & SUSD6 & 93 & CSF3 & 126 & SERPINE1 \\
\hline 28 & PLA2G2A1 & 61 & $K A T 2 B$ & 94 & TNFAIP31 & 127 & FOSB2 \\
\hline 29 & SERPINB2 & 62 & GYPA & 95 & CXCL11 & 128 & EGRI1 \\
\hline 30 & ZBTB162 & 63 & $S G K 11$ & 96 & CXCL61 & 129 & FOS1 \\
\hline 31 & HВМЗ & 64 & KRT5 & 97 & CXCL81 & 130 & ZВТВ161 \\
\hline 32 & HEMGNI & 65 & YODI & 98 & PLA2G2A & 131 & $H B M 2$ \\
\hline 33 & SNCA2 & 66 & $P I P 4 K 2 A$ & 99 & SELE & 132 & RPS4Y1 \\
\hline
\end{tabular}

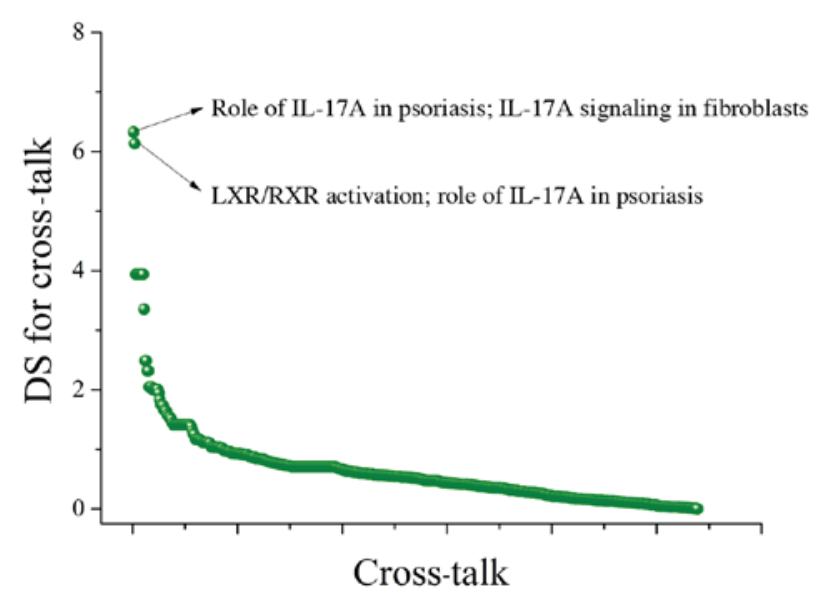

Figure 1. DS was assigned to any pair of DEPs or cross-talk by comparing their gene expression levels. In consequence, 609 cross-talk among 137 DEPs were identified, and the DS distribution are illustrated. DEP, differentially expressed pathway; DS, computing discriminating score. psoriasis (AUC=0.857), and the pair of pathways LXR/RXR activation and hepatic fibrosis/hepatic stellate cell activation (AUC=0.812).

Hub cross-talk. Herein, we divided 20 BPD samples and 30 normal controls of total 54 samples in the gene expression data into two sets according to the ratio of 6:4. Among the 10 seed cross-talk, we uncovered that 3 cross-talk were repeated many times. The pair of pathways role of IL-17F in allergic inflammatory airway diseases and role of IL-17A in psoriasis was repeated 29 times. The pair of pathways role of IL-17A in psoriasis and IL-17A signaling in fibroblasts was repeated 28 times. The pair of pathways IL-17A signaling in airway cells and role of hypercytokinemia/hyperchemokinemia in the pathogenesis of influenza was repeated 28 times. Hence we defined these three cross-talk as hub cross-talk for BPD patients, which might play more crucial roles in the progression of BPD samples than the others. 
Table II. Differentially expressed pathways (DEPs) with DEG number $\geq 4$.

\begin{tabular}{llcc}
\hline DEPs & P-value & $\begin{array}{c}\text { Number of genes } \\
\text { in pathway }\end{array}$ & $\begin{array}{c}\text { Number of } \\
\text { DEGs }\end{array}$ \\
\hline Glucocorticoid receptor signaling & $7.11 \times 10^{-7}$ & 255 & 6 \\
Granulocyte adhesion and diapedesis & $1.55 \times 10^{-6}$ & 163 & 5 \\
Role of IL-17A in psoriasis & $5.96 \times 10^{-13}$ & 13 & 5 \\
Glucocorticoid receptor signaling & $5.32 \times 10^{-6}$ & 255 & 5 \\
Role of IL-17A in arthritis & $2.24 \times 10^{-8}$ & 54 & 5 \\
IL-17A signaling in airway cells & $4.54 \times 10^{-8}$ & 63 & 5 \\
Glucocorticoid receptor signaling & $4.70 \times 10^{-5}$ & 255 & 5 \\
Role of IL-17A in arthritis & $6.74 \times 10^{-7}$ & 54 & 4 \\
IL-17A signaling in airway cells & $1.18 \times 10^{-6}$ & 63 & 4 \\
Agranulocyte adhesion and diapedesis & $5.89 \times 10^{-5}$ & 173 & 4 \\
Role of IL-17A in arthritis & $2.72 \times 10^{-7}$ & 54 & 4 \\
IL-17A signaling in airway cells & $4.78 \times 10^{-7}$ & 63 & 4 \\
Glucocorticoid receptor signaling & $2.25 \times 10^{-5}$ & 255 & 4 \\
Atherosclerosis signaling & $6.60 \times 10^{-8}$ & 119 & 4 \\
Role of IL-17A in psoriasis & $2.63 \times 10^{-9}$ & 13 & 4 \\
Granulocyte adhesion and diapedesis & $1.07 \times 10^{-4}$ & 163 & 4 \\
Agranulocyte adhesion and diapedesis & $1.36 \times 10^{-4}$ & 173 & 4 \\
\hline
\end{tabular}

Table III. Seed cross-talk with AUC value for random forest (RF) classification.

\begin{tabular}{clr}
\hline ID & \multicolumn{1}{c}{ Cross-talk } & AUC \\
\hline 1 & Acute phase response signaling; hepatic fibrosis/hepatic stellate cell activation & 0.915 \\
2 & Role of IL-17F in allergic inflammatory airway diseases; role of IL-17A in psoriasis & 0.857 \\
3 & LXR/RXR activation; hepatic fibrosis/hepatic stellate cell activation & 0.812 \\
4 & LXR/RXR activation; acute phase response signaling & 0.799 \\
5 & Role of IL-17A in psoriasis; IL-17A signaling in fibroblasts & 0.790 \\
6 & IL-17A signaling in airway cells; role of hypercytokinemia/hyperchemokinemia & 0.786 \\
7 & in the pathogenesis of influenza & 0.786 \\
8 & IL-17 signaling; differential regulation of cytokine production & 0.782 \\
9 & in intestinal epithelial cells by IL-17A and IL-17F & 0.782 \\
10 & Role of IL-17F in allergic inflammatory airway diseases; atherosclerosis signaling & 0.778 \\
\hline
\end{tabular}

\section{Discussion}

In the present study, we investigated hub cross-talk of DEPs for BPD samples by integrating a series of bioinformatics analyses, identification of DEGs, extraction of DEPs, exploration of seed cross-talk and investigation of hub cross-talk. Although the scenario might be intuitive, the biological functions and activities of DEPs have not been well studies $(17,18)$. In order to increase the confidence and feasibility of our study, we implemented the MCCV to screen the hub cross-talk $(10,19)$.

Consequently, a total of 3 hub cross-talk for premature newborns with BPD were gained. IL-17F is a cy tokine that shares sequence similarity with IL-17 and is expressed by activated
T cells (20). It inhibits angiogenesis of endothelial cells and induces endothelial cells to produce IL-2, TGFB1/TGFB, and signaling and function in inflammation (21). The involvement of IL-17A/IL-17F in inflammatory immune responses and host defense mechanisms, and the correlation between IL-17A/IL-17F with other cytokines has been reviewed previously $(22,23)$. The review indicated that IL-17A and IL-17F possibly work together in human disease. Coincidently, in our results, the hub cross-talk (role of IL-17F in allergic inflammatory airway diseases and role of IL-17A in psoriasis) also revealed this phenomenon in BPD development.

IL-17A has been demonstrated to take vital part in allergic airway inflammation, and promote inflammation via inducing various proinflammatory cytokines and chemokines, recruiting 
neutrophils, increasing antibody production, and activating T cells (24). We found that IL-17A was correlated to all of the three hub cross-talk of BPD infants, including the pair of pathways role of IL-17F in allergic inflammatory airway diseases and role of IL-17A in psoriasis; the pair of pathways role of IL-17A in psoriasis and IL-17A signaling in fibroblasts; the pair of pathways IL-17A signaling in airway cells and role of hypercytokinemia/hyperchemokinemia in the pathogenesis of influenza. Importantly, IL-17A has been reported to exhibit a vital role in the development of pulmonary fibrosis, suggesting it is a potential treatment target for inflammation-induced fibrosis (25). Taken together, we inferred that the three hub cross-talk closely correlated to BPD.

In summary, 3 hub cross-talk among DEPs were identified in our study, which might give insight into revealing the molecular mechanism underlying the premature newborns with BPD.

\section{Acknowledgements}

Not applicable.

\section{Funding}

No funding was received.

\section{Availability of data and materials}

The datasets used in this study are available from the corresponding author on reasonable request.

\section{Authors' contributions}

CW, RP and BZ conceived the study. MC and GC acquired, and analyzed the data. CW wrote the manuscript. RP, GC and MX explained the results and revised the manuscript. All authors read and approved the final manuscript.

\section{Ethics approval and consent to participate}

Not applicable.

\section{Patient consent for publication}

Not applicable.

\section{Competing interests}

The authors declare that they have no competing interests.

\section{References}

1. Bancalari E and Claure N: Bronchopulmonary dysplasia: Definitions and epidemiology. In: Bronchopulmonary Dysplasia. Bhandari V (ed). Springer International Publishing, Switzerland, pp167-182, 2016.

2. Smith VC, Zupancic JA, McCormick MC, Croen LA, Greene J, Escobar GJ and Richardson DK: Rehospitalization in the first year of life among infants with bronchopulmonary dysplasia. J Pediatr 144: 799-803, 2004.

3. Bhandari A and Panitch H: An update on the post-NICU discharge management of bronchopulmonary dysplasia. Semin Perinatol 42: 471-477, 2018.
4. Watterberg KL, Demers LM, Scott SM and Murphy S: Chorioamnionitis and early lung inflammation in infants in whom bronchopulmonary dysplasia develops. Pediatrics 97: 210-215, 1996.

5. Glaser K and Speer CP: Pre and postnatal inflammation in the pathogenesis of bronchopulmonary dysplasia. In: Bronchopulmonary Dysplasia. Bhandari V (ed). Springer International Publishing, Switzerland, pp55-77, 2016.

6. Gadhia MM, Cutter GR, Abman SH and Kinsella JP: Effects of early inhaled nitric oxide therapy and vitamin A supplementation on the risk for bronchopulmonary dysplasia in premature newborns with respiratory failure. J Pediatr 164: 744-748, 2014.

7. Lavoie PM: Genetics of bronchopulmonary dysplasia. In: Bronchopulmonary Dysplasia. Bhandari V (ed). Springer International Publishing, Switzerland, pp109-127, 2016.

8. Aksamitiene E, Kiyatkin AB and Kholodenko BN: Cross-talk between mitogenic Ras/MAPK and survival PI3K/Akt pathways: A fine balance. Biochem Soc Trans 40: 139-146, 2012.

9. Bernards R: A missing link in genotype-directed cancer therapy. Cell 151: 465-468, 2012.

10. Colaprico A, Cava C, Bertoli G, Bontempi G and Castiglioni I: Integrative analysis with Monte Carlo cross-validation reveals miRNAs regulating pathways cross-talk in aggressive breast cancer. Biomed Res Int 2015: 831314, 2015.

11. Cohen J, Van Marter LJ, Sun Y, Allred E, Leviton A and Kohane IS: Perturbation of gene expression of the chromatin remodeling pathway in premature newborns at risk for bronchopulmonary dysplasia. Genome Biol 8: R210, 2007.

12. Smyth GK: Linear models and empirical bayes methods for assessing differential expression in microarray experiments. Stat Appl Genet Mol Biol 3: Article3, 2004.

13. Bogdan M, Ghosh JK and Tokdar ST: A comparison of the Benjamini-Hochberg procedure with some Bayesian rules for multiple testing. In: Beyond Parametrics in Interdisciplinary Research: Festschrift in Honor of Professor Pranab K. Sen. Vol 1. Institute of Mathematical Statistics, Beachwood, OH, pp211-230, 2008.

14. Routledge R: Fisher's exact test. In: Encyclopedia of Biostatistics. John Wiley Publishing, New York, NY, 2005.

15. Liaw A and Wiener M: Classification and regression by randomForest. R News 2: 18-22, 2002.

16. Xu QS and Liang YZ: Monte Carlo cross validation. Chemom Intell Lab Syst 56: 1-11, 2001.

17. Donato M, Xu Z, Tomoiaga A, Granneman JG, Mackenzie RG, Bao R, Than NG, Westfall PH, Romero R and Draghici S: Analysis and correction of cross-talk effects in pathway analysis. Genome Res 23: 1885-1893, 2013.

18. Yang H, Cheng $\mathrm{C}$ and Zhang W: Average rank-based score to measure deregulation of molecular pathway gene sets. PLoS One 6: e27579, 2011.

19. Xu QS, Liang YZ and Du YP: Monte Carlo cross-validation for selecting a model and estimating the prediction error in multivariate calibration. J Chemometr 18: 112-120, 2004.

20. Besnard AG, Sabat R, Dumoutier L, Renauld JC, Willart M, Lambrecht B, Teixeira MM, Charron S, Fick L, Erard F, et al: Dual role of IL-22 in allergic airway inflammation and its cross-talk with IL-17A. Am J Respir Crit Care Med 183: 1153-1163, 2011.

21. Chang SH and Dong C: IL-17F: Regulation, signaling and function in inflammation. Cytokine 46: 7-11, 2009.

22. Jin W and Dong C: IL-17 cytokines in immunity and inflammation. Emerg Microbes Infect 2: e60, 2013.

23. Puel A, Döffinger R, Natividad A, Chrabieh M, BarcenasMorales G, Picard C, Cobat A, Ouachée-Chardin M, Toulon A, Bustamante J, et al: Autoantibodies against IL-17A, IL-17F, and IL-22 in patients with chronic mucocutaneous candidiasis and autoimmune polyendocrine syndrome type I. J Exp Med 207: 291-297, 2010.

24. Iwakura Y, Nakae S, Saijo S and Ishigame H: The roles of IL-17A in inflammatory immune responses and host defense against pathogens. Immunol Rev 226: 57-79, 2008.

25. Wilson MS, Madala SK, Ramalingam TR, Gochuico BR, Rosas IO, Cheever AW and Wynn TA: Bleomycin and IL-1 $\beta$-mediated pulmonary fibrosis is IL-17A dependent. J Exp Med 207: 535-552, 2010.

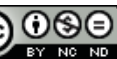

This work is licensed under a Creative Commons Attribution-NonCommercial-NoDerivatives 4.0 International (CC BY-NC-ND 4.0) License. 